\title{
PLANIFICACIÓN Y DESARROLLO DE CIUDADES INTELIGENTES UTILIZANDO HERRAMIENTAS “BIM": UN ENFOQUE INTEGRAL DE GESTIÓN
}

\author{
PLANNING AND DEVELOPMENT OF SMART CITIES BY MEANS OF 'BIM' \\ TOOLS: A COMPREHENSIVE MANAGEMENT APPROACH
}

\author{
Carlos M. Chang ${ }^{1}$, Mario A. Vélez Canchanya ${ }^{2}$
}

RECEPCIÓN: 24 DE AGOSTO DE 2021

ACEPTACIÓN: 10 DE SETIEMBRE DE 2021

\begin{abstract}
RESUMEN
Las ciudades se han vuelto mucho más complejas, y las agencias públicas están enfrentando retos cada vez mayores para brindar un servicio de manera eficiente e inclusivo a toda la comunidad. Las ciudades dependen en gran medida de las tecnologías para una gestión integrada de los sistemas de transporte, energía, seguridad, recursos hídricos, primeros auxilios, y cadena de suministros por mencionar algunos.

Una solución de ciudad inteligente busca la coordinación efectiva de todos estos sistemas, considerando como criterio común el desarrollo sostenible de la ciudad y la calidad de vida de sus habitantes. Esto solo puede lograrse aunando esfuerzos para optimizar el uso de los recursos disponibles (agua, gas, electricidad, uso de la tierra) para maximizar la eficiencia de la infraestructura existente mediante la implementación de sistemas de gestión eficientes.

El concepto de Ciudades Inteligentes es de carácter interdisciplinario y presenta una nueva forma de gestionar la ciudad con la ayuda de los conocimientos y tecnologías de avanzada. Dentro de este concepto, es necesario modelar los diversos escenarios que pueden ocurrir en la ciudad, analizarlos, y buscar la mejor respuesta para las situaciones planteadas. En este contexto, el Building Information Modelling (BIM) es una herramienta que puede mejorar la colaboración y la comunicación entre las partes involucradas en el proceso de gestión de la infraestructura urbana. La integración de diversas disciplinas y la simulación de diferentes situaciones en el BIM puede reducir incertidumbres en la toma de decisiones.
\end{abstract}

Palabras clave: Ciudad Inteligente, BIM, Gestión de Infraestructura.

\begin{abstract}
Cities have become much more complex, and public agencies are facing increasing challenges in efficiently and inclusive service to the entire community. Cities are highly dependent on technologies for an integrated management of transportation, energy, security, water resources, first aid, and supply chain systems to name a few.

A smart city solution seeks the effective coordination of all these systems, considering as common criteria the sustainable development of the city and the quality of life of its inhabitants. This can only be achieved by joining efforts to optimize the use of available resources (water, gas, electricity, land use) to maximize the efficiency of the existing infrastructure through the implementation of efficient management systems.

The Smart Cities concept is interdisciplinary in nature and presents a new way of managing the city with the help of available knowledge and advanced technologies. Within this concept, it is necessary to model the various scenarios that can occur in the city, analyze them, and seek the best answer for the situations raised. In this context, Building Information Modeling (BIM) is a tool that can improve collaboration and communication between the parties involved in the urban infrastructure management process. The integration of various disciplines and the simulation of different situations in BIM can reduce uncertainties in decision making.
\end{abstract}

Keywords: Smart City, BIM, Infrastructure Management.

\footnotetext{
1 Profesor Asociado del Departamento de Ingeniería Civil y Medio Ambiente de Florida International University (FIU), U.S.A.

2 Consultor de Gerenciamiento de Proyectos, Mario Vélez Project Management, Perú.
} 


\section{INTRODUCCIÓN}

La expresión «Ciudad Inteligente» apareció en la década de 1990 y varias definiciones han descrito los diferentes aspectos que las caracteriza. Los elementos comunes en todas estas definiciones son que las ciudades inteligentes utilizan el término "Tecnología de la Información y la Comunicación” (TIC) con la finalidad que los ciudadanos participen en los procesos de gestión con el objeto de mejorar los sistemas de servicio que brinda la ciudad.

La planificación y el desarrollo de Ciudades Inteligentes requiere de la implementación de tecnologías modernas para la interacción eficiente de subsistemas multidimensionales en diversas áreas como son transporte, seguridad ciudadana, energía, gestión de edificaciones, entre otros. Estos subsistemas interconectados deben brindar los servicios necesarios para mejorar la calidad de vida de los ciudadanos al maximizar el uso de los recursos disponibles.

Para el desarrollo de Ciudades Inteligentes es necesario establecer objetivos genéricos o específicos. Los objetivos genéricos apoyan la identidad de un área o estructura urbana determinada, es decir, su propia historia, cultura, medio ambiente o estética. Los objetivos específicos son relacionados a la optimización del consumo de energía, la mejora de la calidad del aire, la reducción del ruido, y la regulación de los sistemas de transporte por citar algunos ejemplos [1].

Con respecto a la tecnología, la gestión de Ciudades Inteligentes requiere establecer una red de sensores con detectores físicos, cámaras para recolectar información y generar imágenes espaciales que faciliten la gestión en tiempo real. Ejemplos de las aplicaciones son mapas de seguridad ciudadana, mapas de temperatura, mapas de emisión de gases. El uso de tecnologías de Ciudad Inteligente resulta en una reducción de costos con una infraestructura urbana resiliente que permita una experiencia de calidad de vida mejorada.

El desarrollo de Ciudades Inteligentes es de carácter interdisciplinario y representa una nueva forma de gestionar la ciudad con la ayuda de los conocimientos y tecnologías de avanzada. Las Ciudades Inteligentes promueven el desarrollo de una red de conocimiento que sirva de soporte para el desarrollo económico y social, enfatizando la integración de las redes tecnológicas y el entorno construido para una mayor interacción de las personas en plataformas tecnológicas interconectadas. Dentro de este concepto general están los componentes de las TIC que son aplicados a áreas urbanas. Las ciudades inteligentes se definen por el nivel de desarrollo de los sistemas urbanos de servicio y por el componente humano-social, mientras que las TIC se definen por los componentes de hardware y software.

Una de las TIC de mayor potencial para su implementación en el desarrollo de ciudades inteligentes es el Building Information Modeling (BIM). El BIM describe el proceso de creación de modelos de construcción digital y el proceso de funcionamiento y mantenimiento mediante el uso e integración a lo largo de todo el ciclo de vida de un proyecto [2]. Las herramientas digitales, como el diseño asistido por ordenador (CAD) y la ingeniería asistida por ordenador (CAE), se han utilizado para diseñar, construir, y operar la infraestructura [3]. El uso de la información recopilada en cada fase del ciclo de vida de un proyecto de infraestructura es difícil y complejo [2]. De esta manera, en la fase de diseño conceptual, las alternativas de diseño son evaluadas visualizando la información geométrica y geográfica para analizar las relaciones espaciales. Las opciones de diseño se optimizan con información adicional de costos de mano de obra y materiales. En la fase de construcción, se simula el proceso de construcción y se supervisa el progreso durante la ejecución. En BIM, todos los datos desde la fase de diseño conceptual a la fase de construcción se recopilan y almacenan en una base de datos visual que es virtual. Cualquier cambio realizado en el modelo se refleja inmediatamente en toda la base de datos [4]. Estos datos digitales se pueden utilizar para operar y mantener actualizada la información necesaria para la gestión durante todo el ciclo de vida del proyecto de infraestructura [2]. Los datos completos del proyecto se pueden utilizar para el diseño preliminar de nuevos proyectos. 
Sin embargo, los componentes tecnológicos y herramientas de soporte son insuficientes por sí solos para el desarrollo de una ciudad inteligente. Es fundamental considerar los resultados deseados al invertir en estas tecnologías. Las ciudades inteligentes deben ser dinámicas y mostrar la capacidad de innovar, reorganizarse, y adaptarse a condiciones que son cambiantes. Las ciudades inteligentes son lugares donde toda la ciudadanía está comprometida con quienes toman decisiones y empoderada para resolver problemas que se presenten. Las ciudades inteligentes deben generar entornos más saludables, vecindarios más seguros, y operaciones más eficientes. En última instancia el grado de evolución de estas características son las que definen el nivel de desarrollo de una ciudad inteligente.

\section{MATERIALES Y MÉTODOS}

Hay muchas tendencias que han llevado al auge de las ciudades inteligentes, una de las cuales comienza con la rápida urbanización de la población mundial. La migración de lo rural a lo urbano comenzó con el inicio de la revolución agrícola e industrial a fines del siglo XVIII. En la actualidad, más del 50 por ciento de la población mundial vive en ciudades. Para el 2050, se proyecta que casi el 70 por ciento de la población mundial, lo que equivale a más de seis mil millones de personas, vivirá en zonas urbanas [5]. Es esta masa crítica de personas las que han establecido las bases para el surgimiento de las ciudades inteligentes.

Debe también tenerse en consideración que con el surgimiento del Covid-19 como pandemia en marzo del 2020 [6] se observó una tendencia a regresar a zonas rurales. Uno de los factores que generó este cambio es la crisis socio-económica generada por el Covid-19, puesto que los costos de vida son más altos en la ciudad, el vivir en zonas rurales resulta más económico. Otro factor para esta tendencia migratoria es la modalidad de teletrabajo, en el cual las empresas ahorran gastos operativos [7].

Entre los objetivos de las Naciones Unidas está el desarrollo de ciudades que sean seguras, resilientes, y sostenibles. Otro objetivo es el desarrollo de la industria a través de soluciones innovadoras para la infraestructura. Ambos objetivos están relacionados y requieren de la implementación de tecnología avanzada para realizarlos [8]. En la práctica, la metodología para desarrollar ciudades inteligentes con herramientas de soporte, como la tecnología BIM, involucra varias etapas que son resumidas en las siguientes:

1. Establecer objetivos genéricos y específicos multidimensionales para el desarrollo de las ciudades inteligentes.

2. Analizar el contexto local y de la interdependencia de los factores que influyen en el desarrollo de ciudades inteligentes.

3. Diseñar un modelo conceptual de gestión adaptado a las condiciones locales para planificar el desarrollo de las ciudades inteligentes.

4. Seleccionar herramientas tecnológicas de soporte para la implementación del modelo conceptual de ciudad inteligente.

5. Desarrollar un programa de actividades y presupuesto a corto, mediano, y largo plazo para la implementación del modelo conceptual de ciudad inteligente con el apoyo de herramientas tecnológicas.

6. Implementar el programa de actividades y recopilar información para la evaluación de los resultados.

7. Retroalimentar el modelo de ciudad inteligente en base al análisis de los resultados de la implementación del programa para refinar periódicamente las herramientas de soporte tecnológico. 
En lo que respecta a la metodología debemos resaltar la importancia de entender los aspectos que definen el carácter multidimensional de una ciudad inteligente, así como de adaptarlos al contexto local para poder seleccionar las herramientas tecnológicas apropiadas para implementar el modelo de gestión de una manera eficiente.

A continuación, se describen los aspectos que caracterizan a una ciudad inteligente y que son necesarios para desarrollar el modelo conceptual, luego se explica cómo se integraría la tecnología BIM para brindar el soporte necesario para la implementación del modelo, entendiéndose como un componente esencial para el desarrollo de las ciudades inteligentes.

\section{Aspectos Multidimensionales para el Desarrollo de Ciudades Inteligentes}

Aspectos medio ambientales: Este aspecto destaca la importancia de la «responsabilidad « que tenemos las personas con nuestro entorno natural. Esto ha llevado a una creciente demanda para realizar una transición a un sistema de suministro de energía neutro en carbono. Es decir, reemplazando los combustibles fósiles con soluciones de energía eólica, solar, y geotérmica que sean más sostenibles, y con mayor eficiencia en lo que respecta a nuestro entorno físico (por ejemplo: control climático automatizado, materiales amistosos al medio ambiente utilizados en el ciclo de vida) y modificando el entorno de trabajo (por ejemplo: reuniones virtuales, teletrabajo) para brindar soporte a una economía colaborativa.

Aspectos financieros: La globalización de la economía mundial y el rápido aumento de las exigencias en cuanto a la calidad en el «nivel de vida» en los países en desarrollo han influido en el auge de las ciudades inteligentes. Si bien es cierto que los países desarrollados empujan constantemente las fronteras de la innovación, a menudo se ven obstaculizados o limitados por sistemas de infraestructura obsoletos, salarios más altos, y sistemas sociales con mayor cobertura (por ejemplo: tarifas de seguros, costos de jubilación). Mientras las ciudades de EE. UU. están adaptando las tecnologías de ciudades inteligentes (como por ejemplo la sincronización de señales en las calles), algunas ciudades en los países en desarrollo están desarrollando ciudades inteligentes desde cero. Los aumentos o disminuciones en los precios del petróleo y el gas y la recesión económica afectan el movimiento de bienes, personas, y dinero en todo el mundo, influyendo en el desarrollo de las ciudades inteligentes en todos los países.

Aspectos políticos: Los consumidores socialmente empoderados exigen más transparencia y responsabilidad de los gobiernos municipales y de las organizaciones no gubernamentales. Cambios profundos en el sistema político deberían facilitar la implementación de tecnologías de avanzada y considerar los beneficios económicos, medio ambientales, y sociales.

Aspectos sociales: Los cambios políticos y técnicos han llevado a cambios significativos en nuestra vida social diaria. Una de estas tendencias es buscar el equilibrio entre el trabajo y la vida personal. En un mundo cada vez más interconectado, a través de teléfonos inteligentes móviles, computación basada en herramientas virtuales residentes en la "nube", combinados con una fuerza laboral móvil a través del teletrabajo, ha permitido que los servicios se realicen en cualquier lugar y en cualquier momento. Las expectativas de la sociedad también han exigido una respuesta en tiempo real, y esto significa que más personas sigan trabajando después de que finaliza la jornada laboral tradicional. El «Big Data» y la disponibilidad de datos en la nube también han proporcionado cantidades masivas de «información» que se pueden utilizar para aplicaciones en tiempo real en las ciudades inteligentes. Esta disponibilidad y necesidad de respuesta en «tiempo real» ha empoderado a los ciudadanos que a menudo tienen acceso a datos y servicios de forma gratuita. Al mismo tiempo, estas tendencias han llevado a un aumento de las preocupaciones sobre la privacidad, la seguridad, y la integridad de los datos.

Aspectos tecnológicos: La rápida disminución de los costos de la tecnología de las comunicaciones ha llevado al desarrollo de la internet, «Big Data», y almacenamiento de información en la nube. 
Como resultado, millones de sensores interconectados por software y hardware conectan a la infraestructura con los usuarios. Por ejemplo, se puede utilizar energía solar y sistemas de control integrados en las edificaciones; implementar sistemas de redes de aguas residuales y transmisión de energía de red inteligente; peajes automatizados en los sistemas de transporte; y datos de rutas en tiempo real para el transporte público. Adicionalmente, los aplicativos en los teléfonos inteligentes con el uso de plataformas sociales, reconocimiento de imágenes y de voz hacen viable la disponibilidad a grandes volúmenes de información (Big Data) en tiempo real. Big data es un término que describe el gran volumen de datos, estructurados y no estructurados, que se transmiten de una manera continua. Sin embargo, la importancia no radica en la recolección y almacenamiento de los datos sino en cómo se utiliza esta información. Los macrodatos se pueden analizar para obtener información que conduzca a mejores decisiones y planteamientos estratégicos de gestión.

El uso de Big Data se está volviendo práctica común en las organizaciones para mejorar su eficiencia utilizando estrategias que son el resultado del análisis de los datos recopilados para poder competir, innovar, y capturar valor. El modelo BIM es imprescindible para poder trabajar de manera virtual realizando simulaciones que acompañadas de la herramienta Big Data permiten a las organizaciones tener conciencia plena de todos los recursos que utilizan, otorgándoles la capacidad de responder a cualquier situación de manera rápida y eficiente. A través de un modelo BIM y el uso del Big Data se puede:

- Conocer en tiempo real el uso de los recursos para optimizar la gestión de consumos de energía.

- Optimizar los diseños con el aprendizaje sobre el desempeño de la infraestructura en servicio.

- Predecir el comportamiento, situaciones de riesgo o alerta a los usuarios.

- Supervisar acciones a través de plataformas integradas virtualmente para la trazabilidad o seguimiento de la respuesta de los diferentes agentes de gestión.

- Simular propuestas y evaluar las acciones de gestión operativa antes de implementarlas.

- Brindar los recursos técnicos para registrar y recuperar información de una fuente confiable.

- Integrar sistemas más complejos que son requeridos para el desarrollo y gestión de una ciudad inteligente.

En resumen, una vez que los datos recolectados son transformados en información coherente, ésta podrá utilizarse para la optimización de los sistemas de gestión de infraestructura y para optimizar los diferentes subsistemas que se requieren para el desarrollo integral de las ciudades inteligentes.

\section{Tecnologías BIM para la Planificación, Desarrollo, y Gestión de Ciudades Inteligentes}

La gestión de la infraestructura es la combinación de actividades de planificación, diseño, construcción, y operación en servicio. En el ciclo de vida de la infraestructura existen diferentes niveles de gestión: estratégico, red macro, selección de proyectos, y proyectos individuales. Para coordinar las actividades e integrar de manera eficiente los diferentes niveles de gestión es necesario contar con herramientas tecnológicas de avanzada como el BIM.

El ISO 19650 [9] describe que el Building Information Modelling (BIM) es una representación digital compartida o modelo de información para facilitar los procesos de diseño, construcción y gestión, y de esta manera proporcionar una base confiable para la toma de decisiones.

Varias disciplinas participan en un proyecto desde la etapa preliminar de diseńo hasta la gestión [2]. Así, por ejemplo, un modelo de información en la etapa de construcción es una representación digital completa del proyecto de infraestructura que incluye información de las dimensiones, relaciones espaciales, información geográfica, cantidades y propiedades de elementos de construcción [10]. Información adicional sobre el proyecto de infraestructura incluye el tiempo de ejecución, costo 
de mano de obra, material y equipo, simulando el proceso completo para optimizar el diseño y la construcción en el espacio virtual [10]. Todas las partes involucradas en el proyecto - propietario, diseñador, contratista, subcontratista y proveedor - pueden colaborar para construir el proyecto de forma más precisa y rentable, reducir residuos y optimizar la eficiencia durante el ciclo de vida del proyecto [10].

En la gestión de la infraestructura, la toma de decisiones es esencial. Sin embargo, es un proceso complejo de objetivos múltiples, y diferentes perspectivas que pueden conducir a conclusiones diferentes. El modelo BIM puede mejorar la colaboración y la comunicación entre las partes. La integración de diversas disciplinas y la simulación de diferentes situaciones en el BIM reducirían las incertidumbres en la toma de decisiones.

Con esta finalidad, desde un inicio se debe establecer y describir cómo se empleará el modelo BIM e identificar los datos que se requieren para maximizar su aporte al valor del proyecto de infraestructura. Por ejemplo, el modelo BIM puede emplearse en la evaluación de las alternativas de diseńo, análisis del ciclo de vida, programación de actividades, estimación de costos, selección de materiales, oportunidades de prefabricación y colocación en el sitio.

Las herramientas multidimensionales del BIM pueden clasificarse en categorías de: tres dimensiones (3D), cuatro dimensiones (4D), cinco dimensiones (5D), seis dimensiones (6D) y siete dimensiones (7D). A medida que aumentan las dimensiones, se integran más datos y modelos [11]. La Figura 1 muestra la estructura de BIM multidimensional.

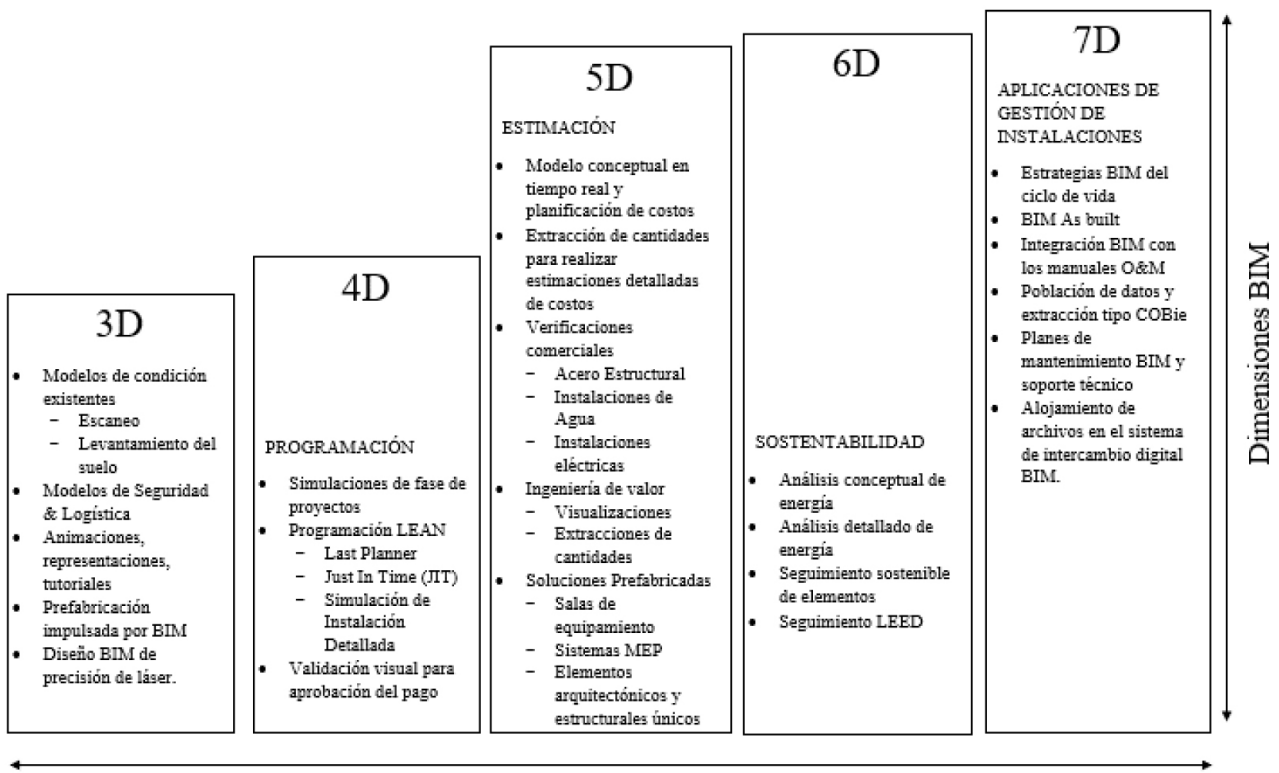

Servicios BIM

Figura 1. Múltiples dimensiones del BIM.

Fuente: Adaptado de 12.https:/lediteca.com/dimensiones-bim-alcance-del-programal

El nivel de madurez del BIM se establecen de acuerdo con el grado de integración del equipo de trabajo alrededor de un modelo único en donde se recopila, almacena y extrae la información. El nivel 0 describe la práctica de trabajo $2 \mathrm{D}$ tradicional basada en el intercambio de dibujos en papel. El nivel 1 incluye la combinación de modelos virtuales 3D y dibujos 2D. Los datos se pueden compartir 
mediante el intercambio de archivos individuales. En el nivel 2, los modelos BIM creados por varias disciplinas se combinan para comprobar si hay conflictos o discrepancias. Todos los datos se pueden administrar y compartir a través de una plataforma central. El nivel 3 describe la integración de modelos BIM para todo el ciclo de vida de un proyecto de infraestructura. Los datos y las descripciones de procesos pueden intercambiarse utilizando estándares ISO. Los datos del proyecto se administran en función de los servicios disponibles en la nube para que se puedan administrar y reutilizar continuamente a lo largo de todo el ciclo de vida del proyecto de infraestructura. La Figura 2 muestra la evolución progresiva de los niveles de madurez del BIM.

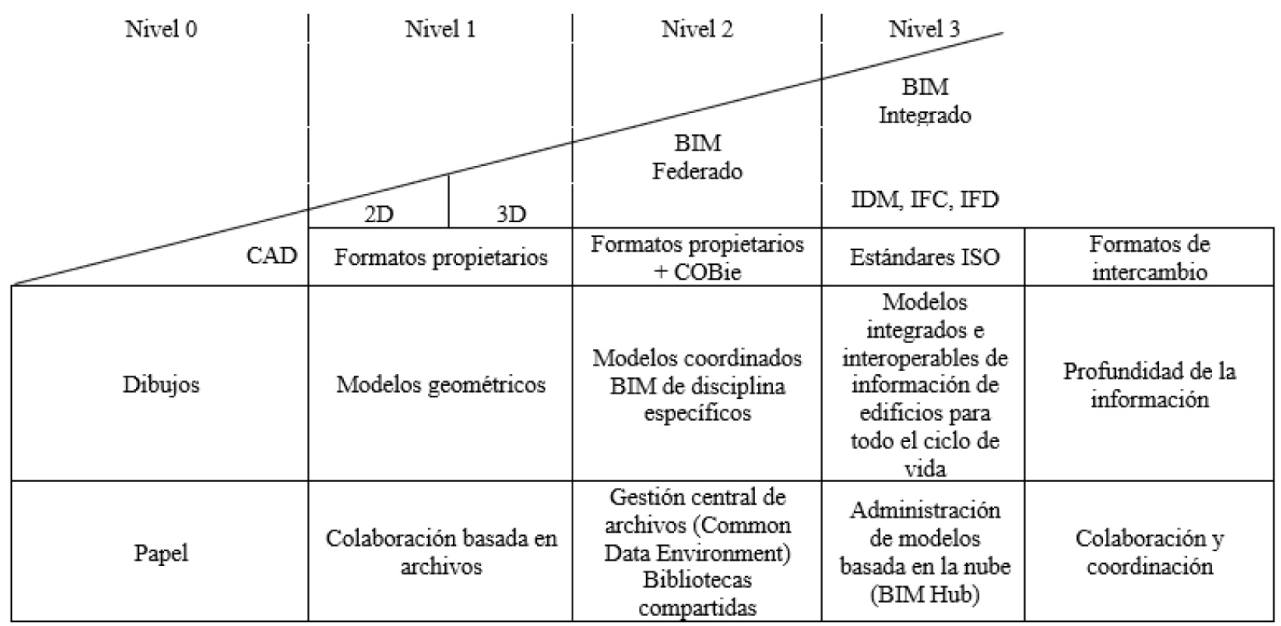

Figura 2. Niveles de Madurez del BIM [2].

Para entender de qué manera el BIM puede ser una herramienta esencial de apoyo en la implementación de las Ciudades Inteligentes, es necesario revisar los principales conceptos, estándares, y aplicaciones utilizadas para gestionar los componentes del BIM, evaluando, de manera directa o indirecta, como contribuirían a la implementación y gestión de una Ciudad Inteligente.

\section{Gestión con BIM}

La serie ISO 19650[9], cuya filosofía general se basa en las normas británicas BS 1192-2 [13] y PAS 1192 [14], resume muy bien el enfoque del trabajo con BIM en donde esta metodología termina siendo un camino por donde transita mucha información, requerida en distintas etapas del ciclo de vida de un proyecto.

En la Figura 3 se puede ver el ciclo completo de generación y procesamiento de la información según el BSI [13], desde la generación del requerimiento (EIR) hasta la puesta en operación del proyecto de infraestructura. En este esquema se puede apreciar el recorrido de la información durante el ciclo de vida del proyecto: necesidad, requerimiento, proceso de licitación, otorgamiento de la buena-pro, diseño, construcción, y operación. También se pueden diferenciar los procesos de información de los procesos de gestión. Por último, se muestran los puntos de decisión críticos para el éxito del proyecto. Los requisitos de información (OIR, PIR, AIR, EIR) así como los modelos de información (PIM, AIM) son descritos en la siguiente sección. 


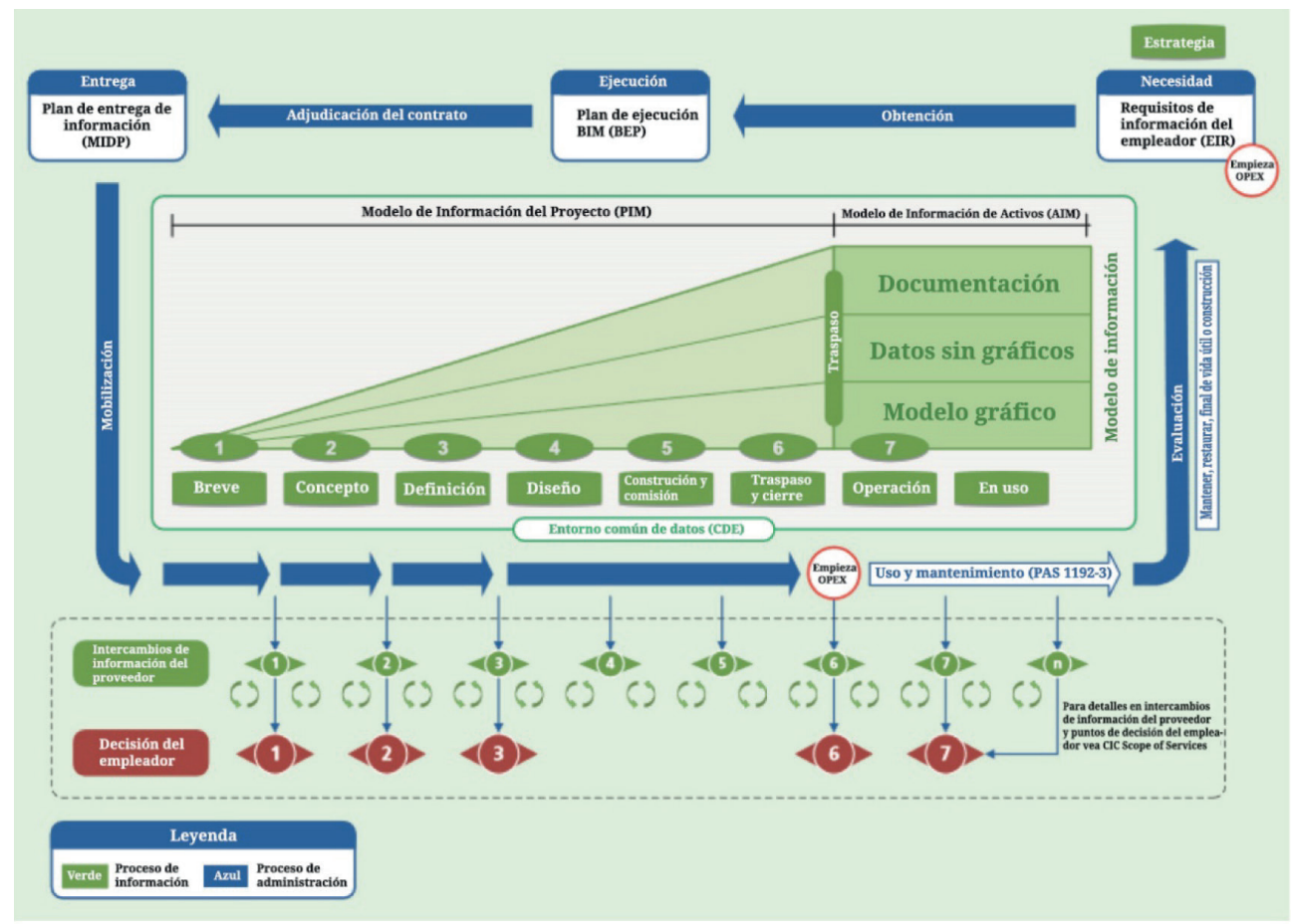

Copyrigth BSI_Mervyn Richards

Figura 3. Ciclo de entrega de información según BSI. [13]

\section{Requisitos de Información para el BIM}

Siendo la información y su tratamiento, los objetivos principales de la metodología BIM, es necesario establecer los requisitos en las distintas etapas del ciclo de vida del proyecto, para acotar de manera eficiente, eficaz y oportuna ambos factores.

Los requisitos de información (ver ISO 19650-1) son un conjunto de especificaciones sobre la información que debe producirse, cuándo debe producirse, su método de producción y su destinatario. Estos requisitos de información son definidos inicialmente por la parte contratante pudiendo ser ampliados estableciendo requisitos particulares de las partes contratadas. Por lo tanto, es importante resaltar que todos los agentes que participan en estos procesos tienen una parte de responsabilidad en la definición de los requisitos que pueden clasificarse como:

- OIR: Requisitos de Información de la Organización relativos a sus objetivos.

- PIR: Requisitos de Información del Proyecto relativos a su desarrollo.

- AIR: Requisitos de Información del Proyecto de Infraestructura relativos a su operación.

- EIR: Requisitos de Intercambio de Información entre dos partes sujetas a una contratación.

Es importante que la parte contratante explique las razones principales por las que se requiere la información solicitada para lograr una mayor comprensión del trabajo colaborativo a realizar. Por otro lado, los modelos de información considerados como entregables pueden clasificarse (ver EN-ISO 19650-1, punto 4) en:

- PIM: Modelo de Información del Proyecto relacionado con la fase de desarrollo. 
- AIM: Modelo de Información del Proyecto de Infraestructura relacionado con la fase de operación.

La Figura 4 define la jerarquía de los requisitos de información con relación a la Organización, la Contratación, y el Modelo entregable.
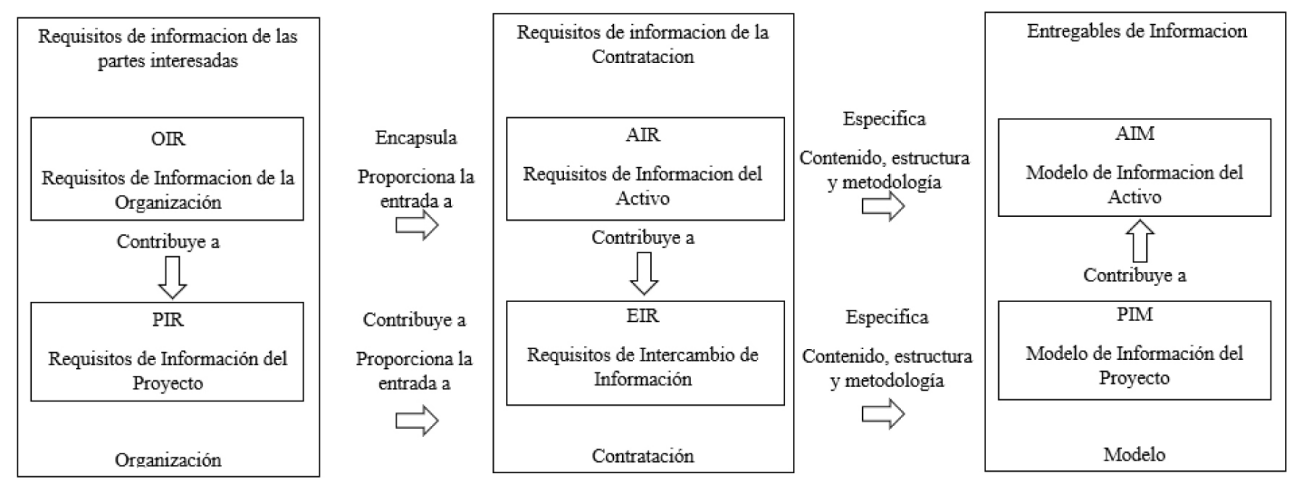

Figura 4. Jerarquía de los requisitos de información según EN-ISO 19650-1. [15]

\section{Entorno BIM, formatos, sistemas de clasificación e interfase con la operación de activos}

Para entender de qué manera el BIM puede ser una herramienta esencial de apoyo en la implementación de las Ciudades Inteligentes, es necesario revisar los principales conceptos, estándares y aplicaciones técnicas utilizadas para gestionar la parte del BIM, que, de manera directa o indirecta, contribuirían a la implementación y gestión de una Ciudad Inteligente.

En primer lugar, se requiere de un entorno o medio virtual, Common Data Environment (CDE) para compartir la información. La información BIM requiere de un formato de comunicación gráfica como por ejemplo el IFC (Industry Foundation Classes).

La información es generada y compartida durante el proceso asignándole algún tipo de clasificación como por ejemplo la de UNIFORMAT ${ }^{\circledR}$, MASTERFORMAT ${ }^{\circ}$ o la de OMNICLASS $®$. Para la transferencia de información a la autoridad que operará el proyecto de infraestructura se utilizan sistemas de clasificación como el COBie.

Éstos y otros aspectos más deberán estar claramente definidos en el Plan de Ejecución BIM (BIM Execution Plan - BEP). Finalmente, para la gestión de la operación del proyecto de infraestructura, se tienen dos grupos principales de sistemas de administración: CAFM y CMMS.

\section{Common Data Environment}

Hablar de BIM es hablar de trabajar de forma colaborativa, y para ello es necesario disponer de un Entorno Común de Datos (CDE por sus siglas en inglés Common Data Environment). El CDE es la fuente acordada de información para cada proyecto de infraestructura, para reunir, gestionar y repartir cada contenedor de información a través de un procedimiento preestablecido.

El modelo de información BIM se convierte en el vehículo que transita por el CDE, recolectando un conjunto de información estructurada (modelos geométricos, propiedades y atributos, programaciones, etc.) e información no estructurada (documentos, imágenes, videoclips, etc.) para facilitar la toma de decisiones. 
IFC

El IFC (*.ifc) es un formato de archivo basado en objetos, desarrollado por [16] buildingSMART International ${ }^{\circledR}$ cuyo objeto principal es el de facilitar la interoperabilidad dentro del sector de la construcción y se utiliza en proyectos de infraestructura basados en el BIM. Es un formato de archivos que permite compartir, intercambiar, información gráfica entre softwares de distintos formatos.

El uso de un CDE y de formatos como el IFC nos permiten compartir información de manera más eficiente y rápida.

\section{Sistemas de clasificación}

Para que exista una eficiente utilización de la información, es necesario emplear los denominados sistemas de clasificación para organizar y recuperar la data. Así se tienen sistemas como el MASTERFORMAT®, UNIFORMAT $®$, OMNICLASS $®$. Todos estos sistemas se mantienen vigentes y son usados alternativamente dependiendo del propósito o etapa del trabajo. La tabla 1 muestra algunos de los principales sistemas de clasificación según el país de origen, las fechas de las primeras y últimas versiones en una revisión hecha en el año 2018.

Tabla 1. Sistemas de clasificación en el mundo (ref. 2018).

\begin{tabular}{|l|l|c|c|}
\hline Sistema & País & Inicio & Última versión \\
\hline UNIFORMAT & Canadá/USA & 1972 & 2010 \\
\hline UNIFORMAT II & USA & 1993 & 2015 \\
\hline MASTERFORMAT & Canadá/USA & 1975 & 2014 \\
\hline UNICLASS & UK & 1997 & 2015 \\
\hline CI/SfB & UK & 1976 & 2002 \\
\hline OMNICLASS & USA & 2006 & 2012 \\
\hline
\end{tabular}

Elaboración propia

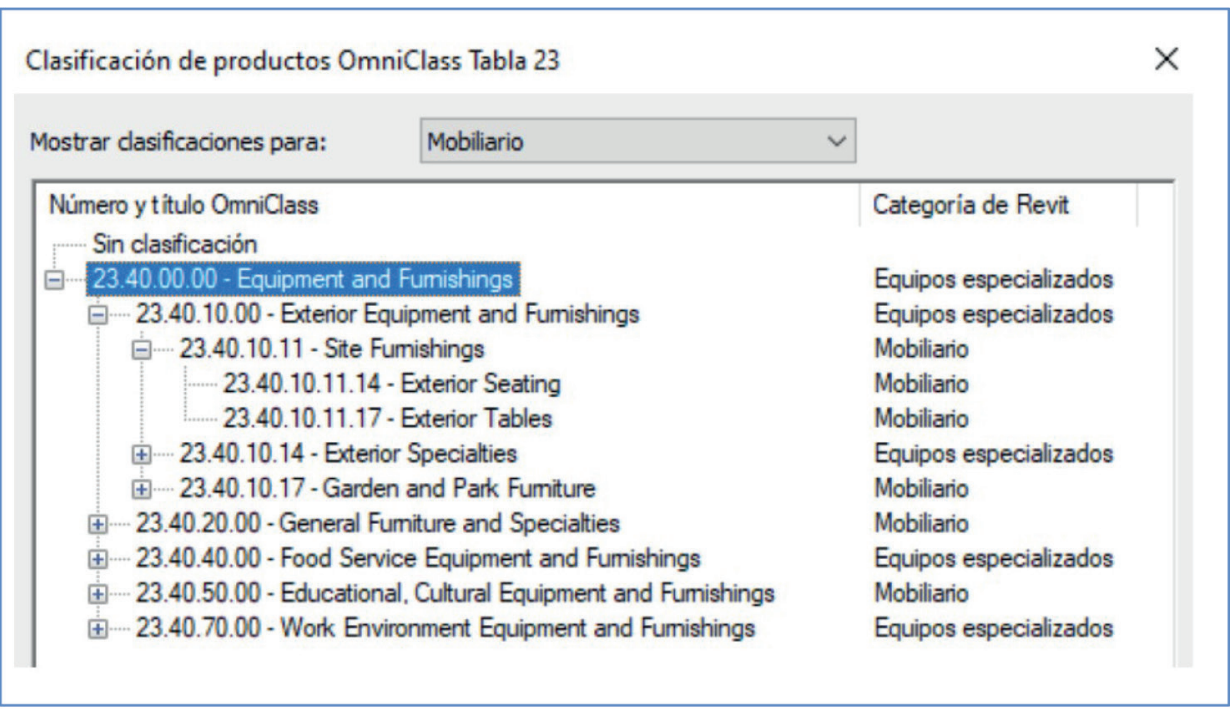

Figura 6. Ejemplo de clasificación OMNICLASS' en software REVIT Autodesk 
Estos sistemas sirven para identificar un elemento gracias a una codificación estandarizada de acuerdo con su función. Por ejemplo, en REVIT Autodesk ${ }^{\circledR}$ un muro de fachada y de tabiquería se realizan con la misma herramienta del software e incluso pertenecen a la misma categoría. Para diferenciarlos se requiere de un sistema de clasificación acorde con su función constructiva y no por la herramienta (polilínea, rectángulo, muro, columna, familia, etc.) con la que la creamos dentro de un determinado software.

Con estas clasificaciones se garantiza que entre las distintas etapas del ciclo de vida del proyecto la información transite identificada con uno de estos códigos garantizando su disponibilidad y rápida ubicación en cualquier momento. La Figura 6 muestra un ejemplo de la clasificación OMNICLASS ${ }$.

MASTERFORMAT ${ }^{\circledR}$ es de origen norteamericano y canadiense, y ha sido desarrollado por el Construction Specifications Institute (CSI), antes de la llegada del BIM e incluso de la existencia de sistemas informáticos. MASTERFORMAT® está enfocado a definir actividades y especificaciones técnicas

UNIFORMAT ${ }^{\circledR}$ organiza la información según el criterio de función. Desarrollado también por CSI, se aplica únicamente a entidades físicas de construcción, es decir, no recoge información relativa a espacios, actividades u otras consideraciones. Está orientado a definir especificaciones constructivas y el análisis de costos.

OMNICLASS ${ }^{\circledR}$ es un sistema de origen norteamericano desarrollado por CSI, y está diseñado para cubrir el ciclo completo de vida del edificio. Es probablemente el sistema de codificación más completo de los existentes. Está hecho pensando en BIM y es organizado por tablas (sistema multitabla) que ordenan entidades dependiendo de la función, la forma, el tipo de actividad, el material, las propiedades, servicios, entre otros. Pretende aglutinar en un sistema único las otras dos clasificaciones más utilizadas que son MASTERFORMAT ${ }^{\circledR}$ y UNIFORMAT®. La Tabla 2 muestra en forma comparativa las características principales de los tres sistemas de clasificación descritos.

Tabla 2. Características de los principales sistemas de clasificación

\begin{tabular}{|c|c|c|c|}
\hline Desarrollador & MASTERFORMAT ${ }^{\circledR}$ & UNIFORMAT ${ }^{\circledR}$ & OMNICLASS ${ }^{\text {CSI/CSC }}$ \\
\hline Basado en & Actividades y entregables & CSI/CSC & CSI \\
\hline Orientación de uso o sistemas & $\begin{array}{c}\text { Organizar } \\
\text { documentación y } \\
\text { especificaciones }\end{array}$ & $\begin{array}{c}\text { Prestema de nueva } \\
\text { generación, hecho } \\
\text { pensando en BIM }\end{array}$ \\
\hline eamposificaciones & $\begin{array}{c}\text { Por facetas, no sigue una } \\
\text { estructura jerárquica }\end{array}$ \\
\hline $\begin{array}{c}\text { Musonado por } \\
\text { diseñadores, porque se } \\
\text { adapta muy bien a las } \\
\text { especificaciones }\end{array}$ & $\begin{array}{c}\text { Puede usarse } \\
\text { también para Project } \\
\text { Management, pero no } \\
\text { es tan detallado como } \\
\text { MASTERFORMAT }\end{array}$ & $\begin{array}{c}\text { Busca ser lo más amplio } \\
\text { posible para que no } \\
\text { ocurra que queden } \\
\text { elementos sin categoría }\end{array}$ \\
\hline NO directamente & SI & SI \\
\hline
\end{tabular}

Elaboración propia

En el futuro, se entiende que deberá haber cierta convergencia en los sistemas de clasificación, sobre todo con la implementación de iniciativas privadas como el OpenBIM y el 16 buildingSMART International ${ }^{\circledR}$ 


\section{COBie ${ }^{\circledR}$}

COBie ${ }^{\circledR}$ es un formato de clasificación de la información que está específicamente orientado a la etapa en que se termina de construir el proyecto y se utiliza para entregar información a la organización que va a gestionar el proyecto de infraestructura.

Conforme un proyecto de infraestructura va avanzando en su ciclo de vida, se genera más metadata. Gran parte de esta información no se requerirá en etapas posteriores, ni sirve para la etapa de operación del proyecto de infraestructura.

Construction-Operations Building Information Exchange, COBie ${ }^{\circledR}$, consiste en una especificación para la captura e intercambio de información del diseño y construcción, para la fase operación y mantenimiento de un proyecto de infraestructura de aquellos elementos que poseen una necesidad de revisión periódica. COBie ${ }^{\circledR}$ ordena la información en diferentes hojas de cálculo. Actualmente se implementa $\mathrm{COBie}{ }^{\circledR}$ como un estándar internacional que ha sido adoptado por diferentes iniciativas, entre ellas el buildingSMART International ${ }^{\oplus}[16]$.

COBie ${ }^{\circledR}$, como plantilla de estructuración y formato de datos, es el punto de partida para la definición y cumplimiento de los requisitos de intercambio de información. Una de las grandes ventajas de $\mathrm{COBie}{ }^{\circledR}$ es el creciente apoyo que recibe en la creación de los dos tipos principales de herramientas de gestión: Computer Aided Facility Management (CAFM) y Computerized Maintenance Management Systems (CMMS).

Los sistemas de clasificación UNIFORMAT ${ }^{\circledR}$, MASTERFORMAT $®$, o el OMNICLASS $\AA$, nos permiten organizar, de acuerdo a la función, la información durante la etapa diseño y construcción del proyecto (CAPEX - Capital Expenditure). El COBie ${ }^{\circledR}$ permite la correcta transferencia de la información a la etapa de operación del proyecto de infraestructura para ser utilizada (OPEX Operating Expenditure). Durante la etapa de operación se utilizan sistemas de gestión de proyectos de infraestructura que se agrupan en dos categorías:

- CAFM (Computer Aided Facility Management)

- CMMS (Computerized Maintenance Management System)

El software CAFM sirve para la administración automatizada de instalaciones y bienes raíces. El software CAFM se integra con múltiples plataformas y ayuda a los administradores de instalaciones a planificar y realizar un seguimiento de cómo se utilizan las instalaciones. Los Facility Manager (FM) pueden usar el software CAFM para programar reparaciones y mantenimiento preventivo, monitorear las órdenes de trabajo y cumplir con los estándares exigidos.

El software CMMS es un componente del software CAFM que está centrado en el mantenimiento. Este software utiliza una única plataforma y tiene una base de datos de las operaciones de mantenimiento de una organización para brindar visibilidad de las decisiones de mantenimiento y las órdenes de trabajo. Los sistemas CAFM interactúan con los sistemas CMMS, al igual que con otras tecnologías como son los sistemas de diseño asistido por computadora (CAD) y los modelos de información de construcción (BIM). Los administradores de instalaciones pueden obtener visibilidad de los edificios de la empresa, cómo se utilizan, el movimiento de componentes de la infraestructura y cómo se relacionan luego de esta integración.

La Figura 7 muestra la interacción de los conceptos y herramientas antes descritas, como parte de un trabajo colaborativo en BIM. 


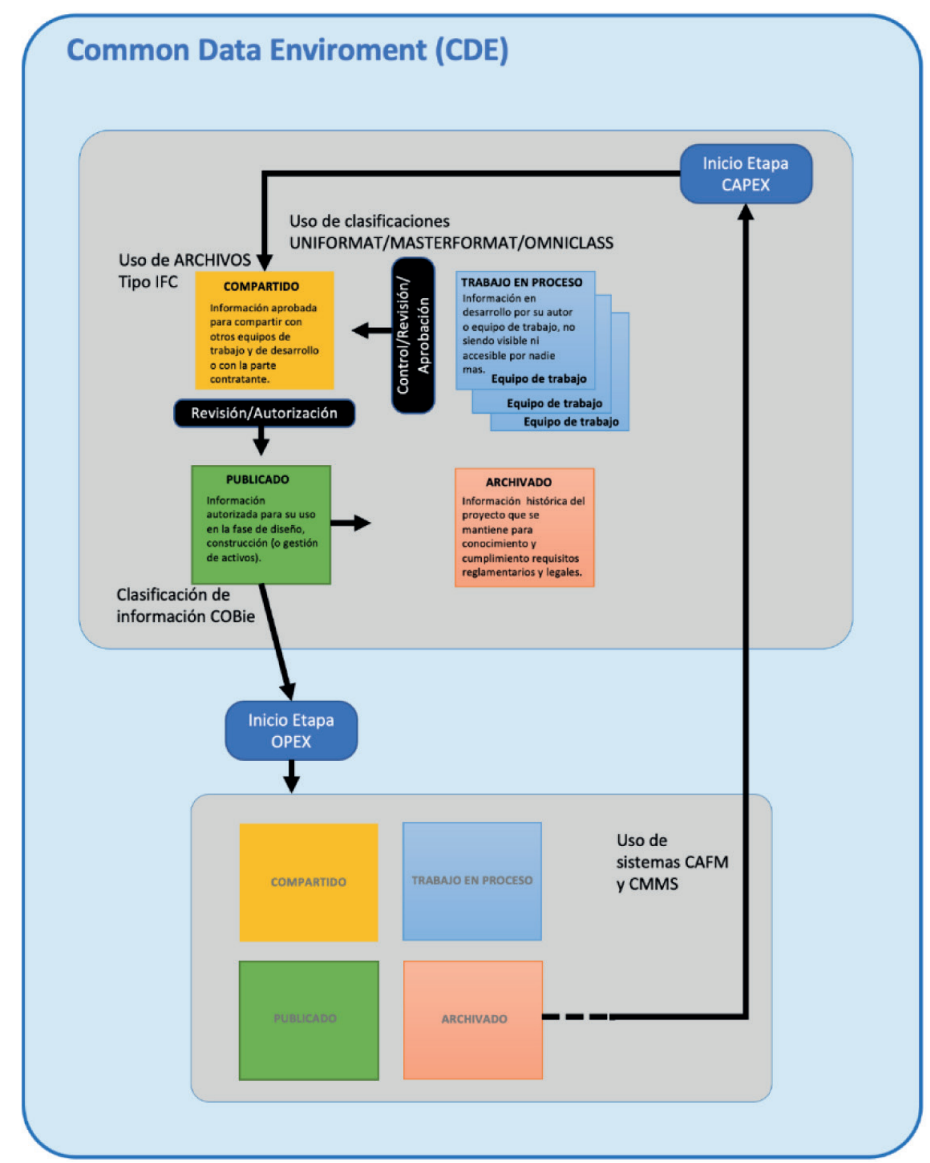

Figura 7. Esquema general del CDE (Common Data Environment).

Elaboración propia.

\section{RESULTADOS Y DISCUSIÓN}

La correcta gestión del BIM y el adecuado uso de los aspectos técnicos que lo integran permitirán ofrecer un modelo de información BIM, sólido, coherente y útil, para la gestión y operación del proyecto de infraestructura. Contar con un modelo BIM consistente, coordinado desde las etapas más tempranas del ciclo de vida del proyecto entre todos los entes interesados (cliente, diseñador, constructor, gerente de operación) constituye un aspecto fundamental para que el proyecto de infraestructura pueda incorporarse como pieza contributiva en el contexto de una Ciudad Inteligente. El propio modelo de información constituye en sí mismo un aporte para el desarrollo de una Ciudad Inteligente.

De esta manera, los resultados esperados de una gestión integral para el desarrollo de ciudades inteligentes utilizando herramientas BIM son los siguientes:

- Una definición clara de la información que necesita el propietario del proyecto de infraestructura, así como de los métodos, procesos, plazos y protocolos de desarrollo y verificación de esta información para contribuir a lograr los objetivos genéricos y específicos multidimensionales de desarrollo de las ciudades inteligentes. 
- Qué la cantidad y calidad de la información desarrollada sea suficiente para satisfacer las necesidades definidas en el modelo conceptual adaptado a las condiciones locales para contribuir al desarrollo de las ciudades inteligentes.

- Transferencia eficiente y efectiva de información entre los diferentes agentes que participan en cada etapa del ciclo de vida del proyecto de infraestructura, especialmente entre la fase de desarrollo y la fase de gestión.

Entre los resultados del uso del BIM está la generación de un gemelo digital que se actualiza en forma dinámica a lo largo del ciclo de vida del proyecto, y que además de almacenar gran cantidad de información permite hacer simulaciones en las etapas de planificación, diseńo, construcción, y gestión. Un gemelo digital es una réplica digital de una entidad física. El gemelo digital se refiere a una réplica digital de activos físicos procesos, personas, lugares, sistemas y dispositivos que pueden utilizarse para diversos fines. La representación digital proporciona los elementos y la dinámica de cómo funciona y se desenvuelve un dispositivo como parte del Internet de las cosas (IoT) durante todo el ciclo de vida.

Entre las características del gemelo digital se enfatiza en primer lugar la conexión entre el modelo físico y el modelo virtual correspondiente o la contraparte virtual. En segundo lugar, esta conexión se establece generando datos en tiempo real mediante el uso de sensores. Con el modelo digital se pueden estimar costos y analizar condiciones existentes durante la planificación. En la etapa de diseño pueden realizarse la detección de interferencias, análisis energético, análisis estructural, evaluación medio ambiental u otros. Durante la etapa de construcción se pueden hacer simulaciones de procesos constructivos, fabricación digital, establecer modelos y documentos As-built. Finalmente, durante la etapa de gestión se podrán establecer e implementar estrategias de mantenimiento y control de riesgos.

A continuación, se presentan ejemplos de aplicaciones del modelo BIM en proyectos y sistemas de gestión para ciudades inteligentes.

\section{Rulesets}

[17] SOLIBRI ${ }^{\circledR}$ es una plataforma de coordinación BIM que permite realizar una serie de trabajos de manera coordinada durante el proceso de diseńo, entre ellos el de detección de conflictos y la verificación del cumplimiento de reglas técnicas (ruleset). Para el adecuado uso de esta aplicación se hace importante haber clasificado la información utilizando alguno de los sistemas antes mencionados (UNIFORMAT ${ }^{\circledR}$, MASTERFORMAT ${ }^{\circledR}$ u OMNICLASS $\left.{ }^{\circledR}\right)$. Por supuesto como parte de su característica de interoperabilidad acepta archivos de extensión IFC.

Actualmente hay varios rulesets disponibles gratuitamente dentro de la plataforma web [17] SOLIBRI $^{\oplus}$, como por ejemplo la de la U.S. General Service Administration GSA, y el capítulo 8.5 del código español, entre otros. Se considera que es una buena alternativa, y su efectividad está supeditada a que esta herramienta sea utilizada en estrecha colaboración con las oficinas urbanísticas de las municipalidades de las ciudades. Softwares de este tipo facilitarán la especificación de una serie de condiciones y requisitos establecidos para la gestión de la ciudad con la finalidad de lograr una eficiente comunicación con la infraestructura existente o por construir.

Un ejemplo de la aplicación de rulesets es el proyecto [18] Apartamentos - Florida (Paradise Gardens). El proyecto consistió en transformar los planos cad 2D a modelos BIM (área construida: $6,120 \mathrm{~m}^{2}$ y 6 niveles) y posteriormente realizar la coordinación espacial de disciplinas y detección de colisiones e incidencias basadas en el código del estado de la Florida. La Figura 8 muestra esquemas del proyecto de Apartamentos y algunos de los modelos de las especialidades que se analizaron. 


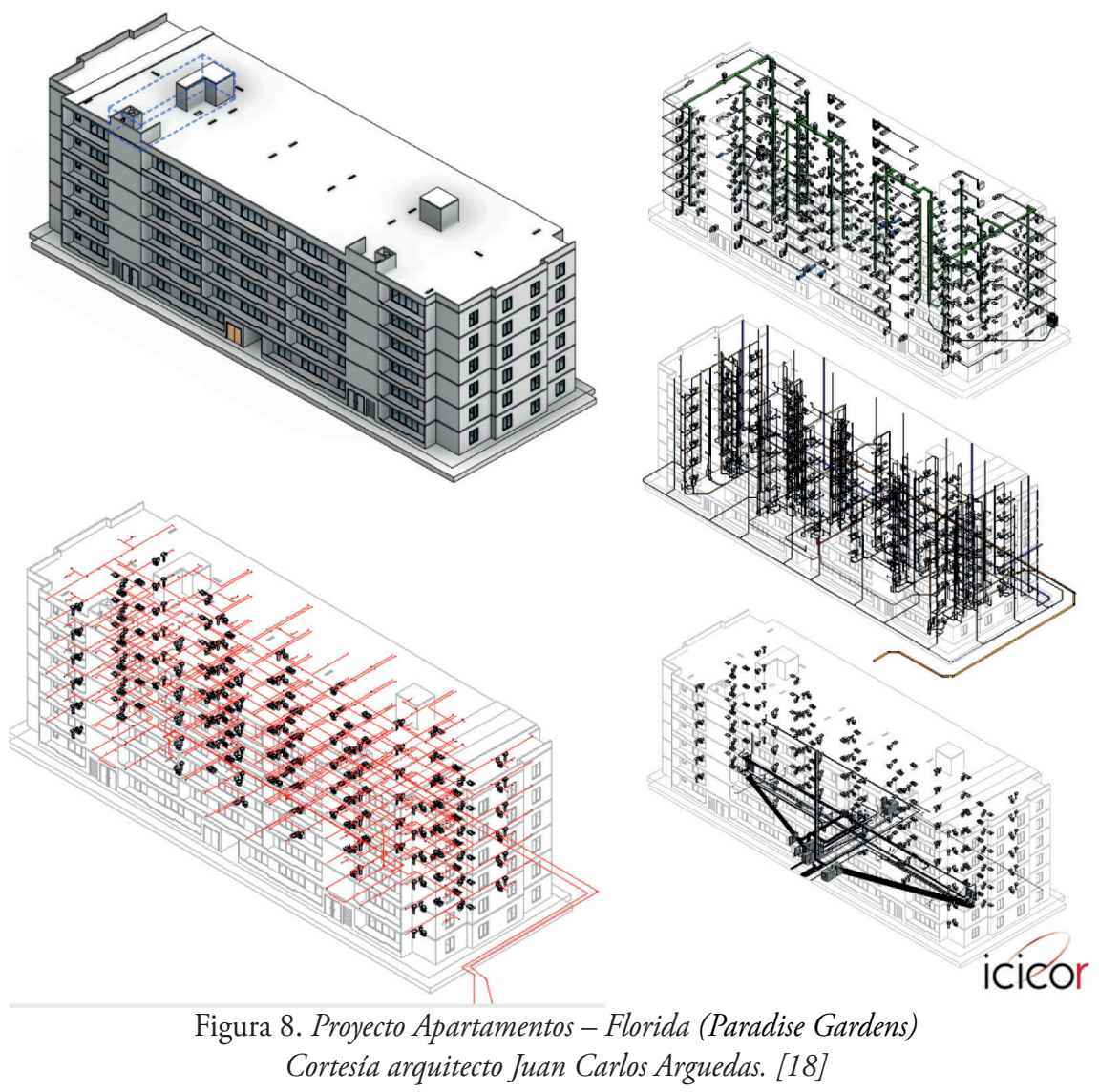

Para ello se cargaron las normas del estado de Florida, como se muestra en la Figura 9, para verificar con el software SOLIBRI ${ }^{\oplus}$ [17] su cumplimiento.

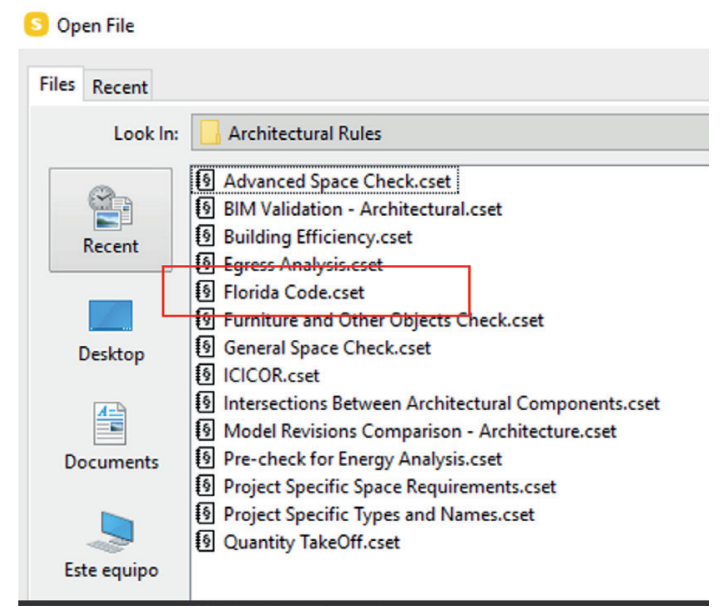

Figura 9. Código de Florida como Ruleset

Cortesía arquitecto. Juan Carlos Arguedas. [18] 
En la Figura 10 se puede ver parte de los reportes de incidencia en donde se resaltan los incumplimientos a las normas estipuladas dentro de la ruleset, los cuales fueron posteriormente corregidos como parte del servicio.
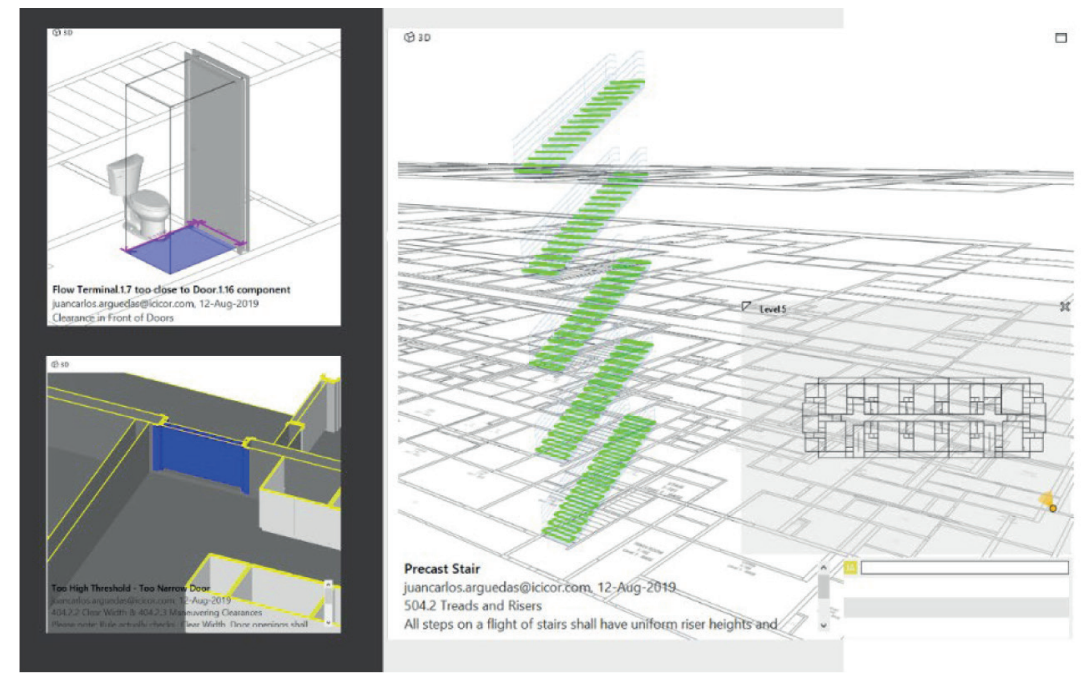

Figura. 10. Reportes de incidencias SOLIBRI ${ }^{\oplus}$ [17]

Cortesía arquitecto Juan Carlos Arguedas. Icicor

Softwares de este tipo permitirán acelerar, automatizar y sobre todo garantizar, la adecuada y aportante incorporación de nuevas edificaciones a una Ciudad Inteligente, cumpliendo normas y estándares locales e internacionales.

\section{Sistema de gestión de activos de fibra óptica GFOAMS}

GFOAMS fue desarrollado por la Universidad de Texas en El Paso en cooperación con la ciudad de El Paso. El objetivo principal de GFOAMS es ayudar a gestionar el sistema de red de fibra óptica. La fibra óptica es el método preferido para las transmisiones de gran volumen de datos debido a su capacidad de banda ancha, seguridad de transferencia de información y no perturbación por señales externas.

GFOAMS tiene información confiable actualizada de las redes de fibra óptica existentes en toda la ciudad. GFOAMS incluye herramientas para facilitar el acceso al inventario y visualizar cualquier detalle específico de la red de conductos de fibra óptica. GFOAMS tiene la capacidad de almacenar y acceder a fotos de fibra óptica tomadas por inspectores de campo. GFOAMS es utilizado por los gerentes con fines de planificación para determinar qué arreglar primero y por los equipos de mantenimiento que realizan inspecciones diarias para evaluar el estado de la fibra óptica, y evitar el mantenimiento diferido. Por ejemplo, en el caso de una ruptura de los conductos y/o cables de fibra óptica en su interior, el gestor de activos puede enviar un equipo de reparación de emergencia con todas las herramientas necesarias para abrir las cajas de conexiones cercanas, reparar los daños y restablecer el servicio [19].

GFOAMS cuenta con numerosas herramientas interactivas para realizar análisis complejos útiles para la planificación a corto y largo plazo. Al mostrar mapas inteligentes con los resultados de los análisis de escenarios de what-if, las múltiples capacidades de GFOAMS han permitido a la ciudad de El 
Paso implementar un sistema de gestión más eficiente ahorrando tiempo y dinero [20]. Dos ejemplos de estas aplicaciones son:

- Análisis de búfer de infraestructura de proximidad: Utilizando las funciones integradas de ArcMap, GFOAMS se utiliza para identificar nuevas rutas en zonas situadas a 100, 500, 1.000 y 2.500 pies de las corridas de conductos existentes. Se pueden establecer diferentes zonas de búfer para analizar opciones al planificar nuevas rutas. Los mapas inteligentes generados a través del análisis de almacenamiento en búfer permiten al responsable de la toma de decisiones visualizar las instalaciones alrededor del sistema de fibra óptica existente, como se muestra en la Figura 11.

- Planificación de nuevos desarrollos de fibra óptica: Con GFOAMS todos los documentos de planificación, dibujos y, específicamente, rutas para futuras redes de fibra óptica se vuelven mucho más simples y eficientes. Además, la visualización del nuevo sistema georreferenciado permite analizar la interactividad entre el sistema de red existente y las adiciones propuestas.

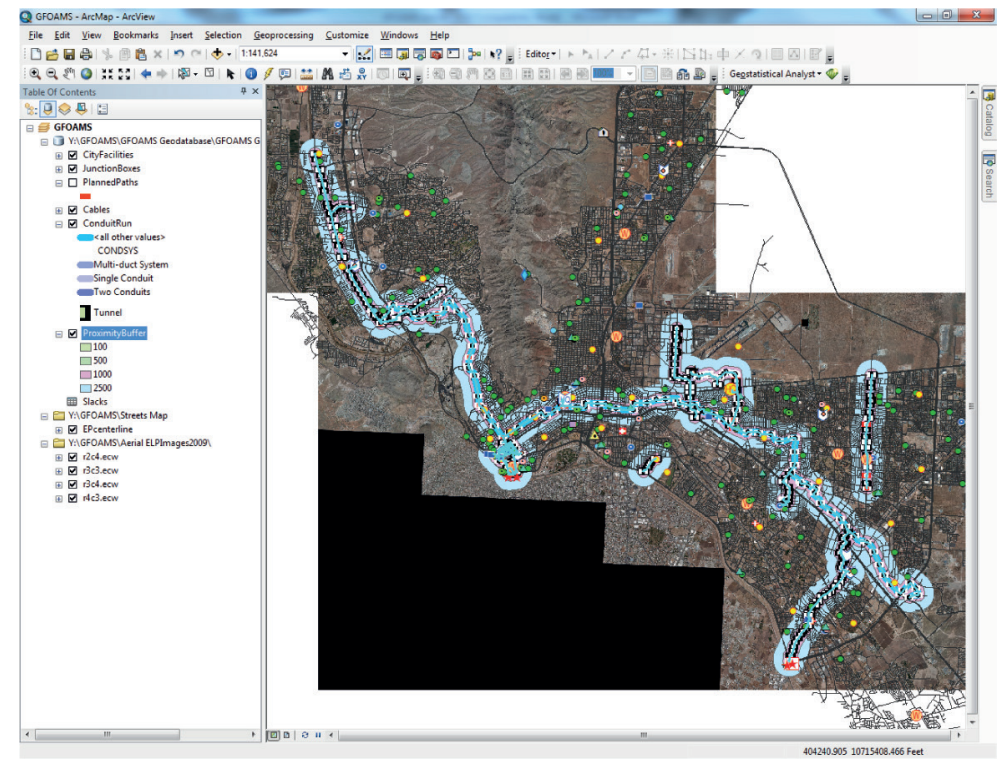

Figura 11. Análisis del búfer de infraestructura de proximidad de las ejecuciones de conductos

\section{ETICA}

Desde una perspectiva de ética, la planificación y desarrollo de ciudades inteligentes utilizando herramientas BIM permitirá un uso más racional de los recursos existentes aplicando de una manera responsable los procesos de ingeniería con la finalidad de contribuir al desarrollo socio-económico sostenible equitativo para todas la personas con respeto, dignidad, e inclusivo anticipándose a las necesidades de la sociedad y utilizando los conocimientos técnicos para mejorar la calidad de vida de todas las personas que viven en una ciudad inteligente.

\section{CONCLUSIONES Y REFLEXIONES FINALES}

1. El desarrollo de las Ciudades Inteligentes es de carácter interdisciplinario y requiere de una combinación de conocimientos y tecnologías innovadoras para el desarrollo económico y social. 
2. La integración de las redes tecnológicas y el entorno físico es esencial y las herramientas de soporte no son suficientes por sí solas para el desarrollo de una ciudad inteligente. Las ciudades inteligentes deben estar en capacidad de innovar, reorganizarse, y adaptarse a condiciones que son dinámicas. La ciudadanía debe participar y comprometerse con quienes toman las decisiones y ser empoderada para resolver los problemas que afectan el desarrollo de entornos más saludables, vecindarios más seguros, y una gestión más eficiente.

3. La tecnología BIM está llamado a desempeñar un rol significativo en el desarrollo de las Ciudades Inteligentes. Definir lo que es un modelo BIM, los objetivos y usos del BIM, las múltiples dimensiones que tiene, así como el nivel de desarrollo (LOD) o el nivel de información (LOI), entre otras características importantes del BIM, permiten entender mejor, con mayor cabalidad, el potencial y sobre todo la mejor oportunidad del uso del BIM como parte de la estrategia de consolidación de una Ciudad Inteligente.

4. El uso de herramientas de soporte como el BIM para una gestión eficiente en las distintas etapas del ciclo de vida de los proyectos de infraestructura involucra la necesidad de un entorno común (CDE). El mejor momento para introducir el BIM en el ciclo de vida de un proyecto es al principio, en el planeamiento. Realmente en esta circunstancia se tendrá la mayor probabilidad de influir positivamente en el comportamiento futuro del activo.

Desde los primeros requerimientos (OIR) hasta la entrega del modelo de información final (AIM) la cual será utilizada en las etapas de operación y mantenimiento, sistemas de clasificación de la información como lo son MASTERFORMAT ${ }^{\circ}$, UNIFORMAT ${ }^{\circ}$, OMNICLASS $\AA$, COBie ${ }^{\circledR}$, y el uso de formatos de archivos como el IFC, permiten que se asegure el flujo eficiente de información entre las distintas etapas de la construcción del proyecto, así como posteriormente también entre los distintos responsables de la operación y mantenimiento del activo resultante; y por lo tanto facilita la puesta a disposición de esa información para la Ciudad Inteligente. Los softwares del tipo CAFM y CMMS permiten gestionar los activos, utilizando como información, la que recibe como parte del modelo del activo resultante (AIM), un gemelo digital.

5. El gemelo digital del Proyecto de infraestructura se convierte en la principal herramienta de colaboración que el BIM aporta a la gestión de una Ciudad Inteligente. Una adecuada y oportuna especificación para el modelo digital, permite que éste se encuentre preparado para servir de nexo, tanto para recibir, como para generar información. Se podrán hacer simulaciones, recolectar grandes volúmenes de data (BIG DATA) que posteriormente se convertirán en conocimiento, revisando en tiempo real la eficiencia de los sistemas para responder eficazmente ante diversas situaciones.

6. El uso de herramientas BIM tipo rulesets permite retroalimentar de manera práctica y automatizada los procesos de gestión de infraestructura en una Ciudad Inteligente. Las lecciones aprendidas, convertidas en normativa de exigencia municipal, permitirán tener edificaciones cada vez más eficientes y se convertirán en elementos aportantes a la ciudad a la que se incorporan. De igual manera, sistema de gestión como el de fibra óptica GFOAMS son herramientas prácticas que facilitan la toma de decisiones y al mismo tiempo las operaciones de mantenimiento.

7. Es fundamental entender los aspectos multidimensionales que definen el carácter de una ciudad inteligente: medio ambiente, financieros, políticos, sociales, tecnológicos Estos aspectos deben ser adaptados al contexto local para luego seleccionar las herramientas tecnológicas apropiadas que permitan implementar un modelo de gestión que sea eficiente. 


\section{REFERENCIAS}

[1] Chang, C., M. Svitek, and T. Hora. Asset Management Systems for A Sustainable Development of Smart Cities. International Conference on Smart Cities, iSMARTI, Corea del Sur, Julio 2019

[2] A. Borrmann, M. König, C. Koch, and J. Beetz. Building Information Modeling - Why? What? How? Chapter 1. Automation in Construction Journal 94, 257-281. 2018

[3] I. Czmoch and A. Pękala. Traditional Design versus BIM Based Design. Procedia Engineering 91, 210-215. 2014.

[4] F. G. B. Blanco, and H. Chen. The Implementation of Building Information Modelling in the United Kingdom by the Transport Industry. Procedia-Social and Behavioral Sciences 138, 510 520. 2014.

[5] United Nations. World Urbanization Prospects. Disponible en: https://population.un.org/wup/ Publications/Files/WUP2018-Report.pdf .2018.

[6] World Health Organization. WHO Director-General's opening remarks at the media briefing on COVID-19. Disponible en: https://www.who.int/director-general/speeches/detail/who-directorgeneral-s-opening-remarks-at-the-media-briefing-on-covid-19---11-march-2020. 2020

[7] A. K. Vazquez. Prevén migración a zonas rurales por pandemia. Diario de Queretaro. Querétaro. Abril 2021. Disponible en: https://www.diariodequeretaro.com.mx/local/preven-migracion-azonas-rurales-por-pandemia-migracion-provincia-crisis-economica-pandemia-noticias-queretaro-6221380.html [Accedido el 29 de abril 2021]

[8] United Nations. The Sustainable Development Goals Report 2020. UN DESA. Disponible en: https://sdgs.un.org/sites/default/files/2020-09/The-Sustainable-Development-GoalsReport-2020.pdf

[9] ISO 19650. Organization and digitization of information about buildings and civil engineering works, including building information modelling (BIM) - Information management using building information modelling. Agosto 2020.

[10] S. Azhar. Building Information Modeling (BIM): Trends, Benefits, Risk, and Challenges for the AEC Industry. Leadership and Management in Engineering 11(3), 241-252. 2011

[11] G. Gourlis and I. Kovacic. Building Information Modelling for Analysis of Energy Efficient Industrial Buildings - A Case Study. Renewable and Sustainable Energy Reviews 68, 953-963. 2017

[12] EDITECA. Dimensiones BIM, el alcance del programa. Disponible en: https://editeca.com/ dimensiones-bim-alcance-del-programa/

[13] M. Richards. BS 1192. Building Information Management. 2010

[14] PAS 1192-2-2013. Specification for information management for the capital/delivery phase of construction projects using building information modeling. 2013

[15] Building Smart Spain. Disponible en: https://www.buildingsmart.es/

[16] Building Smart International. Disponible en: https://www.buildingsmart.org

[17] SOLIBRI Software. Disponible en: https://solution.solibri.com/application/\#!ProductsView

[18] Icicor Costa Rica (CR), 3D As Built (CR), I9 teec (USA) propietario, Apartamentos - Florida (Paradise Gardens), 2019.

[19] L. Quintana, M. Light, C. Chang-Albitres., and R. Aldouri. GIS Fiber Optic Management Systems (GFOAMS). Final Report, University of Texas at El Paso, Junio 2011.

[20] C. Chang-Albitres., L. Quintana, M. C., R. Aldouri and M. Light. Development of a GIS Fiber Optics Asset Management System (GFOAMS). $3^{\text {rd }}$ Work Progress Report, University of Texas at El Paso, Abril 2011. 